\title{
Estimating the prevalence of disability in the community: the influence of sample design and response bias
}

\author{
DAVID LOCKER, RICHARD WIGGINS, YOGA SITTAMPALAM, \\ AND DONALD L. PATRICK \\ From the Department of Community Medicine, St. Thomas's Hospital Medical School, London
}

SUMmaRY An estimate of the prevalence of physical disability in the community based upon a sample survey may be influenced by the sample design and the response to the method of data collection employed. In this paper we describe a postal survey of a sample of households in the London borough of Lambeth and the procedures used for calculating the influence of these factors on the estimate produced. These procedures can be used to adjust the estimate to take account of the relative chance of households falling into the sample and to correct for non-response bias.

A postal survey was employed because it is a cheaper way of collecting small amounts of information than personal interview. ${ }^{1}$ However, the validity of studies using postal surveys may be affected by non-response bias. ${ }^{2}$ Bias arising from non-response has been reported in studies using personal interview. ${ }^{3}$ Since postal surveys require a greater degree of co-operation on the part of the respondent, there is a greater chance that responders and non-responders will differ according to important characteristics.

One solution to the problem of response bias is to maximise response by careful design of the research instrument. ${ }^{2}$ A second method is to use postal or personal interview follow-ups of the non-responders. However, bias may still be a problem if more than $10 \%$ of the sample fail to return the questionnaire or cannot be contacted. In this case, some assessment of the direction and degree of bias is necessary. Because the very nature of the non-response problem usually precludes such an assessment, it is often assumed that missing cases have not distorted the findings. Since this assumption is unacceptable, responders and non-responders should be compared. Where mail surveys have used more than one mailing and/or interview follow-ups, the time trend of bias between successive stages may be used to predict the characteristics of the non-responders. ${ }^{4}$

A postal survey of households rather than individuals was used in the study because past research has shown that it is impractical to send a questionnaire to screen for disability to individuals. ${ }^{5}$ As the electoral register does not contain information on households a sample of individuals must be drawn and used to identify target households. Consequently, the sample design must prevent larger households having a greater chance of falling into the sample.

\section{Method}

The target population for the survey was all adults aged 16 and over living in private households within the London borough of Lambeth at the beginning of 1978. The aim of the sampling design was to achieve a probability sample of individuals from this target population. A household was the sampling unit and it was defined as all those who dwell as a separate living group at an address.

A random start systematic one-in-10 sample was selected from the electoral register. To ensure that small households received the same chance of selection as large households, the firsting principle developed by the Office of Population Censuses and Surveys (OPCS) was used, whereby a surname is selected only if it is the first one within a same-surname sequence in the register. ${ }^{5}$ Otherwise, a surname appearing more than once in the register would have a greater chance of selection. Because this does not equalise the chance of selection of multisurname households, a count was taken of all the surnames listed within a household when the questionnaires were returned. This provides the relative chance of a household appearing in the sample, and is taken to be a 'proxy' count for the number of surnames within a household listed on the register. Any estimate of prevalence of disability 
should strictly take account of these differential probabilities of selection for households, and hence individuals within the households.

The postal questionnaire was developed on the basis of previous surveys of physical handicap in Britain and North America ${ }^{6-8}$ The questionnaire and the method of classifying any named individual as disabled have been reported elsewhere. ${ }^{9}$ The survey was conducted along the lines suggested by the OPCS, ${ }^{5}$ and consisted of a first mailing with reminders posted 16 and 26 days later. Because the response rate to this mail survey was considered to be low, medical students were employed to collect questionnaires from all households from which a response had not been obtained.

\section{Results}

The original sample consisted of 11659 named persons' addresses. Of these, 1294 questionnaires were returned by the Post Office and a further 72 were returned because the named person had died. No reminders were sent to these addresses. The 207 questionnaires sent to individuals resident at addresses that turned out to be old people's homes, hostels, or other institutions were excluded from the study. Of the remaining 10086 , postal returns were received from $\mathbf{7 4 \cdot 1 \%}$. The personal follow-up yielded another $12.5 \%$ to bring the final response to $86.6 \%$. The reasons why a response was not obtained from the remaining households are given in Table 1.

The $\mathbf{8 7 3 5}$ households from which replies were received contained 18744 persons who were known to be aged 16 and over, and who were, therefore, eligible for classification by disability status. Of these persons, $14.6 \%$ were considered to be disabled (Table 2). This is a crude estimate of prevalence derived from unweighted sample data.

As Table 3 shows, $16.6 \%$ of those aged 16 and over listed on questionnaires returned in response to the first mailing were disabled, compared with $11.5 \%$ of those listed on questionnaires returned in response to the third mailing. Of those identified as a result of the personal follow-up, $11 \cdot 5 \%$ were disabled.

Table 1 Response to survey

\begin{tabular}{lrr}
\hline & No. & \multicolumn{1}{c}{$\%$} \\
\hline Questionnaires returned & 7469 & 74.1 \\
$\quad$ By post & 1266 & 12.5 \\
Personal follow-up & 521 & 5.2 \\
Refusals & 813 & 8.0 \\
Not contacted & 17 & 0.2 \\
Discarded: information given could not be used & & \\
& 10086 & 100.0 \\
\hline
\end{tabular}

Table 2 Characteristics of the sample

\begin{tabular}{lrc}
\hline & No. & $\%$ \\
\hline No. of households & 8735 & \\
No. of individuals & 23601 & 100.0 \\
Age & & \\
Unknown & 1110 & 4.7 \\
Under 16 & 3747 & 15.9 \\
16 and over & 18744 & 79.4 \\
Disability status & & \\
$\quad$ Disabled & 2729 & $14.6^{*}$ \\
Non-disabled & 16015 & 85.4 \\
\hline
\end{tabular}

-Unweighted prevalence eatimate.

Table 3 Per cent disabled in each stage of survey (individuals aged 16 and over)

\begin{tabular}{lllllll}
\hline & \multicolumn{2}{l}{ Disabled } & \multicolumn{2}{l}{ Non-disabled } & \multicolumn{1}{l}{ Tocal } \\
\hline 1st mailing & 16.6 & $(1309)$ & 83.4 & $(6588)$ & 100.0 & $(7897)$ \\
2nd mailing & 15.8 & $(595)$ & 84.2 & $(3176)$ & 100.0 & $(3771)$ \\
3rd mailing & 11.5 & $(436)$ & 88.5 & $(3363)$ & 100.0 & $(3799)$ \\
Student follow-up & 11.5 & $(343)$ & 88.5 & $(2645)$ & 100.0 & $(2988)$ \\
& & & & & & \\
Total & 14.6 & $(2683)$ & 85.4 & $(15772)$ & $100.0(18455)$ \\
\hline
\end{tabular}

Excludes 289 individuals for whom stage of return not known $\left(x^{2} p<0.0001\right)$.

ADJUSTING PREVALENCE RATE TO TAKE ACCOUNT OF SAMPLE DESIGN

As noted in the section describing the sample design, the crude prevalence rate of $14.6 \%$ assumes a simple random sample of individuals. This needs to be adjusted by a weighting procedure which takes into account the relative chance of a household appearing in the sample. The procedure used was that of the ratio estimate described by $\operatorname{Cochran}^{10}$; in this case the ratio of the number of disabled to the total number of individuals in the household. The calculation is described in the Appendix. From this an estimated $15.4 \%$ of the target population is disabled with a variance of $0 \cdot 18 \%$.

ESTIMATING THE EFFECT OF NON-RESPONSE It has frequently been reported that non-responders to postal surveys tend to be more similar to late responders than early responders. ${ }^{2}$ Consequently it could be expected that the proportion disabled among the non-responders would be less than that for the responders and that the crude and weighted prevalence estimates would be high. The data show that this is the case in this survey. Overall, $15 \cdot 1 \%$ of the responders to the mail survey were disabled compared with $11.5 \%$ of the non-responders we were able to follow up. The proportion disabled, given full coverage of the study population, can be estimated by means of Hochstim's successive stages model." This model assumes a relationship between the difficulty of obtaining a response, judged by the number of mailings and/or visits necessary to obtain a 
Table 4 Cumulative per cent disabled across successive stages of the survey (individuals aged 16 and over)

\begin{tabular}{lllll}
\hline & Disabled & Non-disabled & Total \\
\hline 1st mailing & $16.6(1309)$ & $83.4(6588)$ & $100.0(7897)$ \\
2nd mailing & $16.3(1904)$ & $83.7(9764)$ & $100.0(11668)$ \\
3rd mailing & $15.1(2340)$ & $84.9(13127)$ & $100.0(15467)$ \\
Student follow-up & $14.6(2683)$ & $85.4(15772)$ & $100.0(18455)$ \\
\hline
\end{tabular}

Excludes 289 individuals for whom stage of return not known.

questionnaire, and a given variable such as disability status. Thus, linear extrapolation beyond the number of stages employed is possible, using the method of least squares. Following Hochstim, we regressed the cumulative per cent disabled against the cumulative per cent of questionnaires returned in order to produce a revised prevalence estimate. This is considered justified where cumulative data show a linear trend. ${ }^{1}$ (Table 4).

Because this procedure requires that the size of study population is known, some additional assumptions were necessary to calculate the number of individuals aged 16 and over living in non-responding households. Both the total number of individuals living in these households and the number eligible for classification by disability status must be estimated. The former requires that a mean household size be specified. Since 8735 responding households generated 23601 individuals this gave a mean of 2.71 members per household. Since $79 \%$ of individuals living in responding households were aged 16 or over, this proportion was applied to the total number of individuals assumed to be living in the non-responding households. The population estimate produced was used to assess the effect of bias on both the crude and the weighted prevalence rates.

Assuming that each individual sampled from the electoral register identified a 'viable' address, that is, an address that was not empty or demolished, there were two groups of households from which no response was received. Firstly, there were those households which may be termed a true non-response group, from whom a questionnaire had not been obtained after the four stages of the survey. This included the refusals, those who could not be contacted during the personal follow-up, and those whose questionnaires were not codable. Secondly, there was the group of households who were not contacted because of inaccuracies in the sampling frame. This included the 1294 addresses from which questionnaires were returned by the Post Office and the 72 where the named person had died. Considering both of these groups as non-responders is somewhat contentious, because the households not covered due to sampling frame inaccuracies were not subject to all stages of the survey. However, we adjusted for all households from which a response was not obtained, because this gives a reasonable indication of the maximum likely bias in the prevalence estimates due to non-response.

As far as the crude prevalence rate is concerned, adjusting for non-response using Hochstim's model produces a revised estimate of $13 \cdot 3 \%$. The estimated proportion disabled among those living in households from which no information was obtained is $\mathbf{9 . 4 \%}$. Calculating the effect of non-response bias on weighted estimates is a complex and time-consuming procedure. In order to apply the successive stages model to the ratio estimate formulae, it is necessary to know how the responding households break down across each stage of the survey according to the number of disabled, the total number of individuals, and the total number of surnames per household. This enables us to make the extrapolations required to adjust the weighted estimate. On the basis of these extrapolations the adjusted weighted prevalence estimate is $14.8 \%$ with a standard error of $0.16 \%$. This is based upon an effective sample size of 10991 households and 24257 persons aged 16 and over.

\section{Discussion}

The response trend reported in this paper is not in agreement with other postal surveys designed to estimate the prevalence of impairment and disability The national survey of impaired and handicapped adults conducted by Harris ${ }^{8}$ found that late returns in response to reminder letters showed no decrease in the proportion of positive replies although the expectation had been that the later respondents were less likely to be impaired. Buckle and Baldwin ${ }^{11}$ followed up non-respondents to a postal questionnaire with personal calls and found no great difference between those who responded to the postal survey and those who were contacted later. However, response trends of the kind we have found have been reported extensively in the literature on postal survey methodology. It is accepted as a general principle that those with an interest in the content of the survey are more likely to reply, so that respondents and non-respondents will have different characteristics.

Because a response trend was observed in the survey reported here, an attempt has been made to assess the direction and degree of likely bias on the crude prevalence estimate and a weighted estimated taking account of the sample design. The weighted estimate is fractionally larger than the crude estimate, although both are judged to be overestimates on the basis of assumptions we have specified regarding total sample size and cumulative 
response trends. The degree of bias appears to be small in percentage terms: a maximum of $1.5 \%$ for the crude rate and $1.0 \%$ for the weighted prevalence rate. The difference is of more significance when estimating numbers of disabled. Given that there are approximately 220000 individuals aged 16 and over living in the borough of Lambeth, a difference of $1 \%$ in the estimated prevalence rate means a difference of 2200 in estimated numbers. This may have important implications if the figures are to be used in planning health and social services.

Where a linear trend is observed in the cumulative proportion of disabled over successive stages of a survey, the lower the response rate the larger the response bias. Where early responders, late responders, and non-responders do not differ, the prevalence rate is independent of the response rate. In the former instance, follow-ups by mail or personal interview are essential. If only one mailing had been used in this study, a crude prevalance rate of $16.6 \%$ would have been produced. This was reduced as returns from successive stages accumulated.

However, the value of mail and personal follow-ups depends upon the extent of likely response bias. The smaller the difference between responders and non-responders, the less the response bias and the less is achieved by improving the response rate. During the collection of data, information is usually not available concerning the characteristics of responders and non-responders, and decisions regarding the necessity of further follow-ups have to be made on the basis of fairly arbitrary assumptions about response trends.

In this survey we assumed that there would be significant differences between responders and non-responders, so that an estimate of prevalence based on a $75 \%$ response rate would be subject to considerable bias. Consequently, we invested considerable resources in obtaining replies from a further $12 \%$ of the sample. It was only after analysis of the data had begun that the validity of this assumption could be ascertained. If information about response trends had been available earlier, a decision about the adequacy of the response rate could have been made in the light of less arbitrary assumptions.

While response trends are usually observed in postal surveys, the studies by Harris ${ }^{6}$ and Buckle and Baldwin $^{11}$ show that this is not invariably the case. It would seem advisable for those conducting future postal surveys of disability in the community to monitor the cumulative proportion of disabled as the replies to successive stages are received. Hochstim's model could then be used to estimate the characteristics of the non-responders in the light of any response trend observed. Knight and Warren ${ }^{12}$

recommend aiming for a response rate of $\mathbf{8 5 \%}$. If no response trend is apparent, or response bias is judged to be insignificant, there is little point in continuing data collection. This is particularly important when financial and other resources are limited.

These suggestions apply only to surveys where the aim is to estimate the prevalence of disability. Follow-ups may be of value even where no response trend is apparent if the objective is to identify risk factors associated with disability. The proportions of disabled among responders and non-responders may be the same but their personal and social characteristics may differ. Nor do the suggestions apply where the aim is case identification or the compilation of registers of the disabled. Here, total rather than sample surveys are necessary. and as complete a coverage as possible of the target population should be the aim.

As the cost of a completed questionnaire increases with the number of stages that need to be employed to obtain a response, the aim should be to obtain the highest possible response to the first mailing. Texts are available which give detailed advice on the conduct of postal surveys and the design of instruments which stimulate interest and therefore response. ${ }^{13}{ }^{14}$ While follow-ups by mail and personal interview may still be necessary, these can be reduced with equivalent savings in time and cost. We have described here a method for estimating likely bias in prevalence estimates due to non-response which can be used to assess the value of such follow-ups in terms of the aims and objectives of the research.

\section{Appendix}

The aim of the analysis was to estimate the proportion of the target population which is disabled such that:

$$
\overline{\mathrm{X}}=\frac{\mathrm{X}_{\mathrm{T}}}{\mathrm{N}_{\mathrm{T}}}
$$

where $\bar{X}=$ proportion disabled

$\mathrm{X}_{\mathrm{T}}=$ number of disabled persons

$\mathbf{N}_{\mathrm{T}}=$ number of individuals sampled

Consider the following summary of the notation necessary for the weighting process:

No. of households

No. of individuals

No. of different surnames on the electoral register

No. of disabled persons

$\begin{array}{cc}\begin{array}{c}\text { Population } \\ \mathrm{M}\end{array} & \begin{array}{c}\text { Sample } \\ \mathrm{m}\end{array} \\ \mathrm{N}_{\mathrm{T}} & \mathrm{n}_{\mathrm{i}}, \mathrm{i}=1, \mathrm{~m} \\ & \\ \mathrm{Z}_{\mathrm{T}} & \mathrm{z}_{\mathrm{i}}, \mathrm{i}=1, \mathrm{~m} \\ \mathrm{X}_{\mathrm{T}} & \mathbf{x}_{\mathbf{i}}, \mathrm{i}=1, \mathrm{~m}\end{array}$


Note that $\mathrm{M}$ and $\mathrm{Z}_{\mathrm{T}}$ are unknown and that it is possible to estimate $\mathrm{NT}_{\mathrm{T}}$. Both the numerator and the denominator (of $\overline{\mathrm{X}}$ ) vary from sampling unit to sampling unit; that is, from household to household. They are considered to be random variables. An estimate which is the ratio of two random variables is known as a ratio estimate. ${ }^{10}$ The estimate of $\overline{\mathrm{X}}$ is then the ratio of two quantities, $\mathrm{X}_{\mathrm{T}}$ and $\mathrm{N}_{\mathrm{T}}$.

$$
\hat{X}_{T}=\frac{M}{m}\left\{\begin{array}{l}
m \\
i=1
\end{array} \frac{x_{i}}{Z_{i}^{\prime}}\right.
$$

where $Z_{i}^{\prime}$ denotes the relative chance of unit $i$ appearing in the sample, that is:

$$
Z_{i}^{\prime}=\frac{Z_{i}}{m_{Z_{i}}} \quad \hat{N}_{T}=\frac{M}{m}\left\{\begin{array}{l}
m \\
i=1
\end{array} \frac{n_{i}}{Z_{i}^{\prime}}\right.
$$

so that

$$
\begin{aligned}
E(\bar{X})=\hat{R}= & \left\{\begin{array}{l}
m \frac{x_{i}}{Z_{i}} \\
\left\{\frac{n_{i}}{Z_{i}}\right.
\end{array}\right.
\end{aligned}
$$

Reprints from Dr. David Locker, Department of Community Medicine, St. Thomas's Hospital Medical School, London SE1 7EH.

\section{References}

${ }^{1}$ Hochstim JR, Athanasopoulos DA. Personal follow-up in a mail survey: its contribution and its cost. Public Opinion Q 1970-71; 34: 68-81.

${ }^{2}$ Scott C. Research on mail surveys. J R Stat Soc 1961; 124: 143-95.

${ }^{3}$ Cox A, Rutter M, Yule B, Quinton D. Bias resulting from missing information: some epidemiological findings. $\mathrm{Br}$ J Prev Soc Med 1977; 31: 131-6.

${ }^{4}$ Hochstim JR. A critical comparison of three strategies of collecting data from households. J Am Stat Assoc 1967; 62: $976-89$.

${ }^{5}$ Harris AI, Head E. Sample surveys in local authority areas. London: OPCS Social Security Division, 1974.

${ }^{6}$ Harris AI. Handicapped and impaired in Great Britain I. London: HMSO, 1971.

${ }^{7}$ Bennett A, Gerrard J, Halil T. Chronic disease and disability in the community $\mathrm{Br}$ Med J 1970; iii: 762-4.

${ }^{8}$ Haber LD. Identifying the disabled: concepts and methods in the measurements of disability. Soc Secur Bull 1978; 30: 17-34.

${ }^{9}$ Patrick D, Wiggins D, Locker D, Green S, Darby S, Horton G. Screening for disability in the London Borough of Lambeth. London: Department of Community Medicine, St. Thomas's Hospital Medical School, 1978.

${ }^{10}$ Cochran WG. Sampling Techniques. New York: John Wiley, 1962.

${ }^{11}$ Buckle JR, Baldwin P. Survey of handicapped and impaired persons aged 75 or over and living alone in the Royal Borough of Kensington and Chelsea. London: Royal Borough of Kensington and Chelsea, 1972.

${ }^{12}$ Knight R, Warren M. Physically disabled people living at home: a study of numbers and needs. London: HMSO, 1978.

${ }^{13}$ Dillman A. Mail and telephone surveys: the total design method. New York: John Wiley, 1978.

${ }^{14}$ Clausen JA, Ford RN. Controlling bias in mail questionnaires. J Am Stat Assoc 1947; 47: 497-511. 